\title{
Analysis of the Needs of Relatives of Severely Ill Patients in ICU
}

\author{
Marinaki Chrisoula ${ }^{*}$ and Papageorgiou Dimitris ${ }^{2}$ \\ ${ }^{1}$ ICU Nurse, Greece \\ ${ }^{2}$ Nursing Department, University of West Attica, Greece \\ *Corresponding author: Marinaki Chrisoula, ICU Nurse, 121 Megalou Alexandrou, 18755 Athens, Greece
}

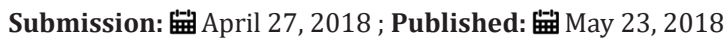

\begin{abstract}
Objective: Analysis of the needs of relatives of seriously ill patients treated in ICU.

Method: Review in the MEDLINE, CINAHL and Cochrane databases of published studies in the last 15 years to reach consensus through an expert panel.

Result: Visiting the patient is one of the main needs of the family. There are several types. Various studies have also been published on about its necessity and what type is more appropriate, as well as the 2017 guidelines. The medical staff should adapt visiting hours according to the needs of relatives and staff. Giving information to relatives is also one of the main needs which should not be omitted. Various published studies prove this and strongly recommend various strategies to improve the process of updating by doctors. The meaning of spirituality is diachronic. According to studies that have been carried out, religious belief is a very important need of relatives and health professionals should always intervene in order to cater for it. The stressful experience of patient's admission to the ICU causes many negative emotions to the relatives and creates the need for psychological support. Numerous studies praise the necessity and interventions for psychological support from health professionals.
\end{abstract}

Conclusion: Health professionals should try to cater for the needs of the family and treat it as a distinct group of people who needs special management, attention, care, update, support and approach.

Keywords: Family needs; Severely ill patients; Intensive care unit; Nursing care

\section{Introduction}

The patient's unexpected admission and hospitalization in the ICU is a stressful situation for both the patient and his family. The patient and the rest of the family are a system of people connected with strong motional ties and if a member is seriously ill, the rest of the members should take on the lead role in care but also in physical and emotional well-being [1,2]. In addition, these members are experiencing needs that are soaring due to the particular environment of ICU, as well as a variety of negative emotions such as anger and despair. Since 1970s, the scientific community has been particularly interested in exploring the needs of the family throughout the patient's hospitalization in the ICU.

Olding et al described five main needs for participation of patients and relatives that have been investigated: visiting hours cater for needs / psychological support, communication / informing, decision making and patient care [3]. The only quantitative research on the needs of families in Greece was conducted in 2010 at the University Hospital of Heraklion, Crete [4]. Numerous studies have been published on the policy and how visiting hours in the ICUs are being implemented, but also on how they should be applied [5-7]. Several studies have been carried out on the need for family informing in overseas hospitals [7-12]. There are many published studies on the need for religious belief of patients and their families [7,13-15]. The need for psychological support has also been studied by several researchers $[6,7,16-20]$.

\section{Discussion}

The only quantitative research on the needs of families in Greece was conducted in 2010 at University Hospital of Heraklion, Crete. Researchers ranked family needs using the CCFNI questionnaire based on their answers. The conclusion was that the need for reassurance is the most important need for relatives, as they considered the question of "answering your questions honestly" as the most important. Also, the conclusion that has been drawn seems to be related to the educational and socio-economic background of the relatives [4]. 


\section{The need for access and proximity}

The main need of the family in ICU is to be close to their relative. To meet this need they only have the visiting hour available to them. There are specific policies for the ICU visiting hours based on the size of the hospital, geographic location, patient traffic and the willingness of health professionals. There are five types of visiting hours:

\section{A. Open or liberal visiting hours.}

There are no restrictions on residence time and frequency of visits. Access to the family is allowed at all times. This type of visit is mainly applied to the pediatric ICU, unlike the adult ICU where it is unusual. Its benefits are related to the reduction of the symptoms of anxiety and depression in the patient and to a much better degree of family satisfaction. On the other hand, the disadvantages are that the workload of the ICU's medical staff may increase and there may be delays in the performance of tasks. Nurses seem to be cautious about this type of visit, despite the benefits [21].

B. Exclusive visiting hours. When it is allowed to visit at any time of the day or night, except for certain specified hours set by the staff.

C. Limited visiting hours. Visits can be made at specific times of the day and with a limited number of visitors at a time [21].

D. Structured visiting hours. This type of visit differs from the limited one only in the duration of relatives' stay.

E. Flexible visiting hours. There is an agreement between patient, relatives and staff about the timetable of the visit [22].

According to the published guidelines, the presence of family in the ICU is very important. Relatives of critically ill patients should be offered flexible access so they can be next to the patients. Also, according to the protocol, staff should provide support to relatives in these difficult hours and enable them to participate in the interdisciplinary decision-making team and attend a recovery with a staff member if they wish to do so.

A further intervention in the guidelines is to take measures for environmental hygiene, to reduce noise in the ICU and to provide family sleep in order to reduce the impact of sleep deprivation [7]. Generally, with regard to the visiting hours, the relatives do not remain in the ICU for a long time since these units are designed as a closed type. Environmental conditions within the ICU should be characterized by high technology, artificial lighting and reduced noise, so as to contribute to the reduction of anxiety.

In a Fumagalli's et al. [5] study, it was observed that in the cardiological ICU, the average visit time was 2.6 hours per day when applying an open visit. They also emphasized that open-time visits to ICU do not increase the risk of septic complications, while, on the contrary, they decrease cardiovascular complications that are probably due to the reduction in anxiety [5]. The policy of closed type visits appears to be accepted by relatives, perhaps because the family wants to allow the patient to rest. However, many authors welcome the fact that in order to respect the rights of the patients and their relatives, as well as to improve the satisfaction of their needs, the adoption of an open or a liberal visiting policy is required.

It has been shown that such interventions help reduce stress among patients and their families and increase confidence in healthcare professionals. A duality between open and closed type visits is evident from the above. According to Vallejo et al. [6], the flexible visit policy is considered to be an average solution designed to meet the needs of the family and the patient focusing mainly on anxiety reduction [6]. Pardavila \& Vivar [10] in their bibliographic review agreed that the main need is detailed information from the staff and immediately after this need comes the need for proximity. They have also come to the conclusion that these needs present the need for written information and flexible working hours [10].

\section{The need for information}

When informing the relatives, health professionals enter into a moral challenge of either giving hope or being completely honest [12]. It is even more difficult to inform when the end of the patient's life approaches. There are ten strategies to improve communication in such cases and these are the following:

A. The doctor should make the first move and approach the relatives. S/he must not wait for them to approach him/her.

B. Then s/he has to introduce herself/himself.

C. Her/His attire and behavior must be appropriate for the occasion. Simple and comprehensible language should be used.

D. Suitable room for discussion.

E. All members of the family should be included in the discussion without someone being disregarded.

F. During the discussion, there should be honesty, courtesy and avoidance of excessive optimism.

G. Processes of repression and isolation, which are not the same as those of care and treatment, should be highlighted.

H. The doctor must be willing to admit that s/he can't have the answers to all issues.

I. The doctor must give the relatives time so to listen to them.

J. Prompting question-asking.

Relatives want their questions to be answered in a comprehensible and honest way. They also want to be informed about changes in the patient's clinical picture as soon as possible [12]. Communication with family members, based on the guidelines, should include frequent interdisciplinary family meetings, structured approaches to doctors' communication, and a written information sheet, especially when the patient is in critical condition. It is also very important that the ICU doctors be 
trained for the family-focused communication segment. All of these contribute to developing relatives' trust towards doctors, reducing conflicts between these two and minimizing the family's feelings of anxiety.

In addition, family education programs and family information sheets should be included as part of clinical care as they have shown beneficial effects in reducing anxiety and depression [7]. The most important tips for improving family care are regular family informing and decision-making sessions, open visiting hours, evaluation of information practices and the teaching of communication skills to ICU health professionals [23]. Studies and interventions related to family sessions are constantly proliferating, especially in USA hospitals. "Intensive communication" is an intervention program that has united the family meeting with the ICF medical staff. Informative brochures are an intervention that has shown very satisfactory results in terms of a better understanding of the patient's condition. In France, the distribution of a family information sheet has already been implemented.

These brochures included information such as:

A. Information about ICU medical staff, for example director's name, supervisor's name, physicians' names.

B. Information about the hospital, such as visiting hours, nearby transport links, useful phone numbers.

C. A diagram of ICU names.

D. The utility of machines that support the patient and

E. Explanation of commonly used medical terms, such as intubation, mechanical ventilation, extrusion, suppression, inhospital infection.

All the above led to a much better understanding of the patient's condition and course, with only a small percentage (3.4\%$6.9 \%$ ) of relatives with insufficient comprehension [12]. Generally, a paternalistic approach to decision-making in ICU at the end of life is prevalent in Europe. Research has shown that in Spain the patient's family was not included in the decision-making process at a rate of $28 \%$, while in France 56-83\%. In Italy, 19\% of health professionals said that the close family had never been involved in such discussions.

Finally, it has been proved that families are not often happy with end-of-life care as a result of inadequate communication. This phenomenon should be addressed, since approaching the family with good communication at the end of a patient's life can make the process easier for all those directly involved [9].

Various studies have been carried out on the need for family information in hospitals abroad. Khalaila [11] emphasized that this need depends on a variety of factors such as gender, age, personality, educational level, marital status, and so on. Typically, relatives want information not only about the course of the disease and about the treatment, but also about practical issues related to the patient's daily life or even financial issues [11].
In their publication, Pochard [8] \& Azoulay [23] developed three models of interactions between relatives and health professionals. Firstly, in the first model there is a family representative who is informed but does not make decisions or participate in physical care. In the second, ICU staff provides care to the patient according to his wishes, just as described by his relatives. Finally, in the third model, the patient's family is involved not only in decision-making but also in physical care [8].

\section{The need for spirituality}

Religion is closely linked to the issue of health, as there is a belief that the disease comes from God and praying helps prevent it. It improves and enhances the ability to deal with the disease, increases social support, while also helps patients to develop a positive image for themselves. This promotes health and fights the disease off in a better way. It is also likely that religion will inhibit unhealthy habits, such as substance abuse and smoking.

Life-threatening illnesses create significant emotional, physical and financial effects on both the patients themselves and their families. Each person reacts in a special and unique way to loss, which may or may not affect his quality of life. Spirituality seems to be a factor that can be associated with a change in the quality of life, as all people have spiritual needs that give purpose and meaning to their lives, whether they believe or not [24]. In two recent studies, McSherry and Ross and Guay et al. [15] report that for some patients the mental needs are more important than the physical ones, as they contribute to a better acceptance and treatment of the disease and hence, to a better quality of life $[13,15]$.

Vachon et al. [14], In 2009, correlated spirituality with 10 dimensions:

A. The purpose of life.

B. The meaning of life.

C. Overcoming the ego.

D. Transcendence with the help of a superior force.

E. Beliefs.

F. Hope.

G. Faith

H. Attitude towards death

I. Reflections on the core values of life.

J. Feelings about social support and society [14].

\section{The need for psychological support}

The patient's family while experiencing the hospitalization of their loved one in the ICU, also experiences mental problems such as anxiety, intense stress and depression. Hospitalization in the ICU creates intense and psychological stress not only in the patients themselves, but sometimes even more in their family environment. This psychological stress has been associated with poor physical 
well-being, but also with premature mortality especially of relatives who have been the caregiver in patients with chronic illnesses. Also, during hospitalization in ICU it has been shown that relatives may pay less attention to their own needs than to the patient's. This is, however, a situation which may affect the health of the relatives themselves, but also their ability to support the patients [18]. According to the Bronfenbrenner ecological model, each family member treats anxiety in a different way in the event of an illness or the admission of a member in the hospital [6].

In 1967, Thomas Holmes and Richard Rahe in an innovative research concluded that serious events in life create stress and intense stress creates illness. In a tailored version of the list created by Holmes and Rahe was the CUSS (Undergraduate Stress Scale), in which the serious illness of a close friend or family member as an event is rated 85. Namely, a person who is experiencing such a situation is much more likely to develop an illness than someone who is not experiencing it. Schmidt et al in their research concluded that the patient's family experience a high rate of anxiety and depression symptoms. Also, in their study, $40 \%$ of the relatives of patients who died exhibited psychiatric illnesses one year after the loss [25].

The World Health Organization (WHO) argues that palliative care should not only focus on improving the quality of life of patients but also that of their families. The current guidelines suggest that family meetings be systematically conducted for all patients, particularly those who have economic or other implications due to their admission to the hospital [18]. Also, according to the guidelines 2017, it is very important to have a psychologist and a social worker in the meetings of the interdisciplinary team and the family to reduce psychological symptoms [7].

The medical staff should take care of the family environment of all patients, but much more of those with a higher risk of death in ICU. Relatives of such patients are confronted and experience intense stress and emotional load, so their care should be an indispensable part of providing holistic care. The intense emotional burden is mainly due to the choice of treatment and decision making for their loved one, which causes psychological symptoms such as anxiety and depression, but also physical symptoms. As a result, the general well-being of all members of the patient's family is influenced [16]. Communication between medical staff and relatives, as well as the assessment of their needs, is of utmost importance as an element of palliative care. This inadequate communication can have major negative effects on psychology due to inadequate supplying of information, mistrust of health care benefits and non-shared decision-making for the patient [20].

In Rusinova's et al. [19] study on psychological need, relatives in the ICU appeared to suffer from symptoms of depression and anxiety. In many countries around the world, symptoms of anxiety appear to be as high as 75\%. Limited communication between ICU staff and patient's family seemed to be associated with a higher incidence of anxiety symptoms. However, this factor can be improved and a goal can be set for preventive intervention. Researchers mention that the average duration of communication between health professionals and relatives should be about 16 minutes. In this way, the psychological needs of the relatives are met and the symptoms of anxiety and depression related to information are addressed. In Eastern European countries, family members estimated that the length of contact with the physician was less than ten minutes and was therefore considered short [19].

It has been shown that limited communication between ICU staff and relatives is associated with a high incidence of symptoms of anxiety and depression. However, this factor can be improved and set as a target for preventive intervention. Researchers say the average duration of communication between health professionals and relatives should be about 16 minutes. In this way, the psychological needs of relatives and the symptoms of anxiety and depression that associated with information are treated [19].

\section{Conclusion}

Visiting is one of the most important family needs and in no way should the family be deprived of it. An adjustment of the conditions and its duration should be made to meet the preferences and needs of relatives and patients in conjunction with the need of the staff to manage the appropriate care of the severely ill. Effective updating provides a variety of benefits to the relatives, such as stress reduction, disposal of confusion, better understanding of the patient's clinical course, and empowerment for active family involvement in decision-making.

Religion improves and enhances the ability to deal with the disease, increases social support, and helps patients develop a positive image for themselves. This promotes health and fights the disease. It is also likely that religion will inhibit unhealthy habits, such as substance abuse and smoking. Finally, the World Health Organization (WHO) argues that palliative care should not only focus on improving the quality of life of the patient but also that of his family.

\section{References}

1. Agard SA and Harderb I (2007) Relatives' experiences in intensive care-Finding a place in a world of uncertainty. Intensive and Critical Care Nursing 23(3): 170-177.

2. Al Mutair AS, Plummer V, Clerehan R, O Brein A (2013) Needs and experiences of intensive care patients' families: a Saudi qualitative study. British Association of Critical Care Nurses 19(3): 135-144.

3. Olding M, McMillan S, Reeves S, Schmitt M, Puntillo K, et al. (2016) Patient and family involvement in adult critical and intensive care settings: a scoping review. Health Expectations 19(6): 1183-1202.

4. Chatzaki M, Klimathianaki M, Anastasaki M, Chatzakis G, Apostolakou E, et al. (2012) Defining the needs of ICU patients families in a suburban/ rural Greek population: a prospective cohort study. Journal of Clinical Nursing 21(13-14): 1831-1839.

5. Fumagalli S, Boncinelli L, Lo Nostro A, Valoti P, Baldereschi G, et al. (2006) Reduced cardiocirculatory complications with unrestrictive visiting policy in an intensive care unit: results from a pilot, randomized trial. Circulation 113(7): 946-952. 
6. Vallejo A, Fernandez D, Gutierrez A, Fernandez M (2016) Analysis of needs of the critically ill relatives and critical care professionals' opinion. Med Intensiva 40(9): 527-540.

7. Davidson J, Aslakson RA, Long AC, Puntillo KA, Kross EK, et al. (2017) Guidelines for Family-Centered Care in the Neonatal, Pediatric and Adult ICU. Critical Care Medicine 45(1): 103-128.

8. Azoulay E, Pochard F (2003) Communication with family members of patients dying in the intensive care unit. Curr Opin Crit Care 9(6): 545550.

9. Gullo A, Besso J, Lumb DP, Williams GF (2009) Intensive and critical care medicine. Springer Verlag Italia. World Federation of Societies of Intensive and Critical Care Medicine, Italy.

10. Pardavila Belio MI, Vivar CG (2012) Needs of the family in the intensive care units: a review of the literature. Enferm Intensiva 23(2): 51-67.

11. Khalaila R (2014) Meeting the needs of patients' families in intensive care units. Nurs Stand 28(43): 37-44.

12. Farahani M, Gaeeni M, Mohammadi N, Seyedfatemy N (2014) Giving information to family members of patients in the intensive care unit: Iranian nurses' ethical approaches. J Med Ethics Hist Med 7: 9.

13. McSherry W and Ross L (2002) Dilemmas of spiritual assessment: considerations for nursing practice. J Adv Nurs 38(5): 479-88.

14. Vachon M, Fillion L and Achille M (2009) A conceptual analysis of spirituality at the end of life. Journal of Palliative Medicine 12(1): 53-59.

15. Guay DMO, Hui D, Parsons HA, Govan K, De la Cruz M, et al. (2011) Spirituality, religiosity, and spiritual pain in advanced cancer patients. Journal of Pain and Symptom Management 41(6): 986-994.

16. McAdam JL, Puntillo K (2009) Symptoms experienced by family members of patients in intensive care units. Am J Crit Care 18(3): 200-209.
17. Needham DM, Davidson J, Cohen H, Hopkins RO, Weinert C, et al. (2012) Improving long-term outcomes after discharge from intensive care unit: report from a stakeholders' conference. Crit Care Med 40(2): 502-509.

18. Choi J, Hoffman LA, Schulz R, Ren D, Donahoe MP, et al. (2013) Health risk behaviors in family caregivers during patients' stay in intensive care units: a pilot analysis. Am J Crit Care 22(1): 41-45.

19. Rusinova K, Kukal J, Simel J, Cerny V (2014) Limited family members/ staff communication in intensive care units in the Czech and Slovak Republics considerably increases anxiety in patients' relatives- the Depress study. Biomed Central 14: 21.

20. Hudson P, Girgis A, Mitchell G, Philip J, Parker D, et al. (2015) Benefits and resource implications of family meetings for hospitalized palliative care patients: research protocol. BMC Palliative Care 14: 73.

21. da Silva Ramos FJ, Fumis RR, Azevedo LC, Schettino G (2013) Perceptions of an open visitation policy by intensive care unit workers. Ann Intensive Care 3(1): 34.

22. Khaleghparast S, Joolaee S, Ghanbari B, Maleki M, Peyrovi H, et al. (2016) A Review of Visiting Policies in Intensive Care Units. Global Journal of Health Science 8(6): 267-276

23. Azoulay E, Chaize M, Kentish Barnes N (2014) Involvement of ICU families in decisions: fine- tuning the partnership. Animals of Intensive Care 4: 37.

24. Nixon AV, Narayanasamy A Penny V (2013) An investigation into the spiritual needs of neuro-oncology patients from a nurse perspective. BMS Nursing 12: 2.

25. Schmidt M, Azoulay E (2012) Having a loved one in the ICU: the forgotten family. Curr Opin Crit Care 18(5): 540-547.
Creative Commons Attribution 4.0

International License

For possible submissions Click Here

\section{Submit Article}

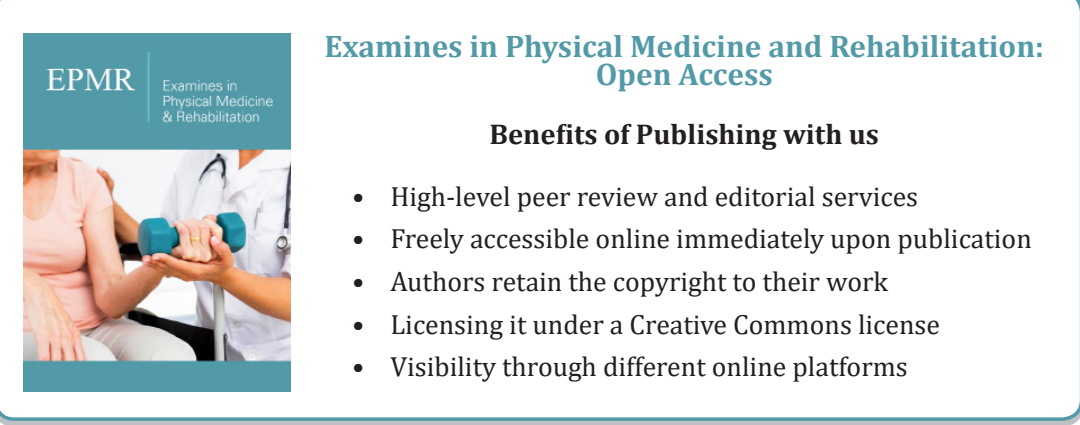

